Письма в ЭЧАЯ. 2006. Т. 3, №7(136). С. 89-95

УдК 621.384.633.5/6

\title{
STATISTICAL PROPERTIES OF THE RADIATION FROM VUV FEL AT DESY (FEMTOSECOND MODE OF OPERATION)
}

\author{
E. L. Saldin, E. A. Schneidmiller, M. V. Yurkov \\ Deutsches Elektronen-Synchrotron (DESY), Hamburg, Germany
}

\begin{abstract}
Present bunch compression scheme at the VUV FEL at DESY is essentially nonlinear and naturally results in a formation of a short high-current leading peak (spike) in the density distribution that produces FEL radiation. The main feature of the considered mode of operation is the production of short, down to $20 \mathrm{fs}$, radiation pulses with GW-level peak power and contrast of $80 \%$.

Система компрессии электронных сгустков для лазера на свободных электронах (ЛСЭ) в DESY является существенно нелинейной, что естественным образом приводит к концентрации заряда в головной части сгустка. Излучение ЛСЭ производится в основном головной частью электронного пучка, которая имеет высокий пиковый ток и короткую длительность. Характерными особенностями такого режима работы ЛСЭ являются короткая, до 20 фс, длительность импульса, гигаваттный уровень выходной мощности и высокий, до $80 \%$, контраст излучения.
\end{abstract}

\section{INTRODUCTION}

The project of the vacuum-ultraviolet free electron laser (VUV FEL) at DESY is realized in two phases. Phase I (1999-2002) served for a proof-of-principle of self-amplified spontaneous emission (SASE) FEL operation and for system tests of the hardware. Phase II of the VUV FEL has been built as an extension of Phase I to shorter wavelengths (down to $6 \mathrm{~nm}$ ) and was used as the first VUV FEL user facility starting in the spring of 2005. The VUV FEL Phase I demonstrated unique femtosecond mode of operation which was not considered at an early design stage of the project $[1,2]$. Thorough analysis has shown that due to nonlinear compression and small local energy spread the short high-current (3 kA) leading peak (spike) in the bunch density distribution was produced by beam formation system. Despite strong collective effects (of which the most critical was the longitudinal space charge after compression) this spike was bright enough to drive FEL process up to the saturation for the wavelengths around $100 \mathrm{~nm}$ [3]. In addition to the possibility for production of high-power femtosecond pulses, this mode of FEL operation demonstrated high stability with respect to drifts of machine parameters. Successful operation of the VUV FEL Phase I in the femtosecond regime encouraged us to extend such a mode of operation for shorter wavelengths. Relevant theoretical study has been performed in [4]. It has been found that the beam formation system of the linac can be tuned for production of bunches with a highpeak-current spike capable of effectively driving the FEL process so that the VUV FEL can safely saturate even at the shortest design wavelength of $6 \mathrm{~nm}$ with a GW level of the peak power in short pulses of $15-50$ fs duration. 
Optimum parameters determined in [4] have been chosen for the commissioning of the VUV FEL. First experimental results obtained at the VUV FEL operating at the radiation wavelength around $30 \mathrm{~nm}$ did show perfect agreement with predictions [5]. Commissioning of the VUV FEL proceeded in parallel with first user experiments. Our contacts with user community did show that planning of future user experiments at the VUV FEL requires more detailed knowledge of the expected statistical properties of the source, and the present paper covers this gap.

\section{RADIATION PROPERTIES}

Operation of the bunch formation system has been studied in detail in [4]. We considered two possible options of operation with a nominal charge of $0.5 \mathrm{nC}$, and with higher charge,

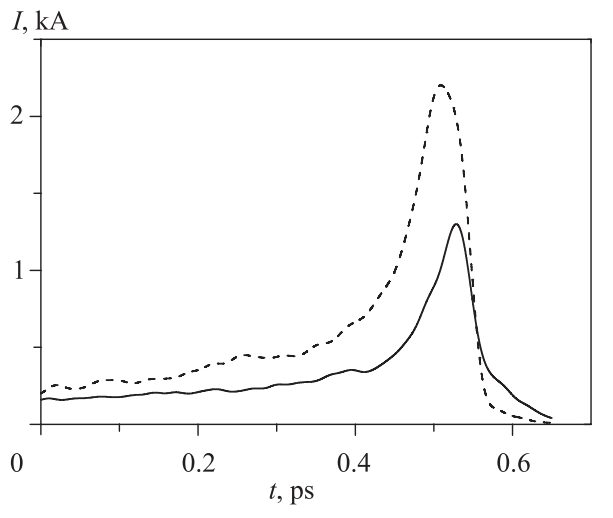

$1 \mathrm{nC}$ (see Fig. 1). Both options can be realized experimentally and provide different modes of the VUV FEL operation in terms of output characteristics of the radiation. Complete set of the electron beam properties at the undulator entrance can be found in [4].

Fig. 1. Current along the electron bunch. Solid and dashed lines correspond to bunch charge of 0.5 and $1 \mathrm{nC}$, respectively. Bunch head is on the right side

Some output characteristics of VUV FEL (energy in the radiation pulse, angular divergence, etc.) have been described in our previous paper [4]. Here we present more detailed features of the radiation related to ultrashort pulse duration. To extract more detailed information about photon beam properties, we performed 500 statistically independent runs with FEL code FAST [6]. Figure 2 shows mean energy in the radiation pulse and rms fluctuations
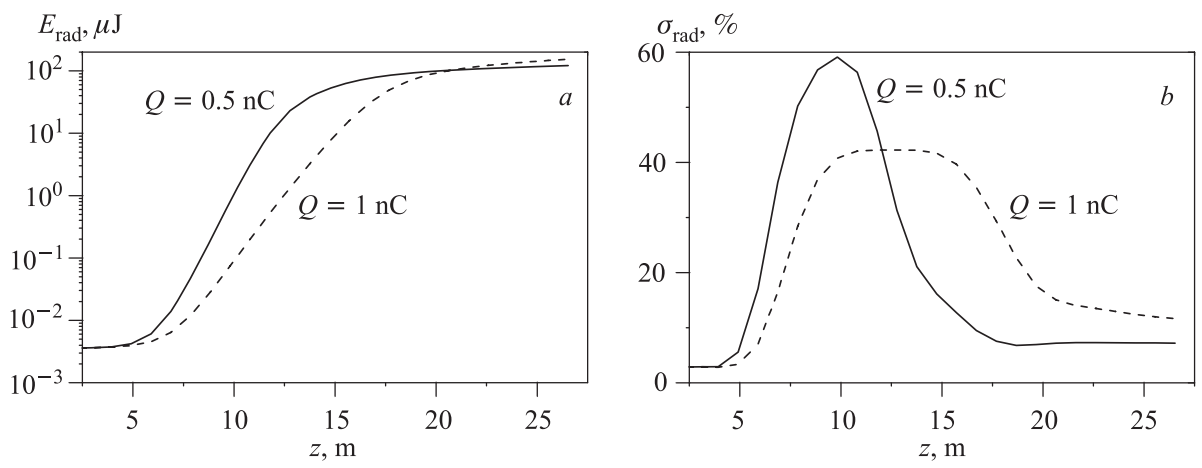

Fig. 2. Expected performance of the VUV FEL at the radiation wavelength of $30 \mathrm{~nm}$. $a$ ) Energy in the radiation pulse versus undulator length. $b$ ) Fluctuations of the energy in the radiation pulse versus undulator length. Solid and dashed lines refer to bunch charge of 0.5 and $1 \mathrm{nC}$, respectively 

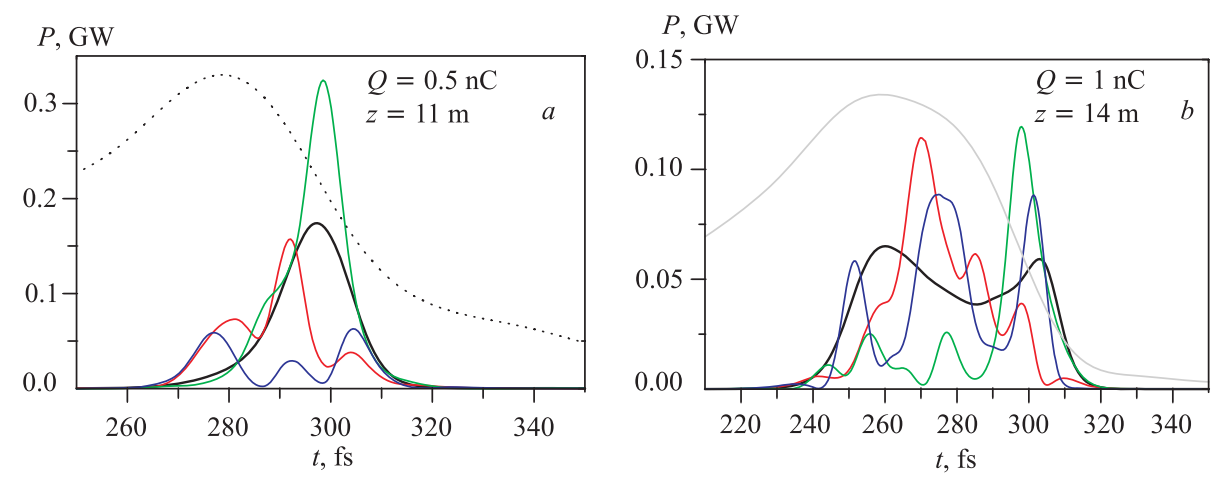

Fig. 3. Radiation power along the bunch for the VUV FEL operating in the linear regime. a) Bunch charge is $0.5 \mathrm{nC}, z=11 \mathrm{~m}$. b) Bunch charge is $1 \mathrm{nC}, z=14 \mathrm{~m}$. Thin curves show single shots. Bold curves show averaged profiles. Grey curves show profile of electron bunch
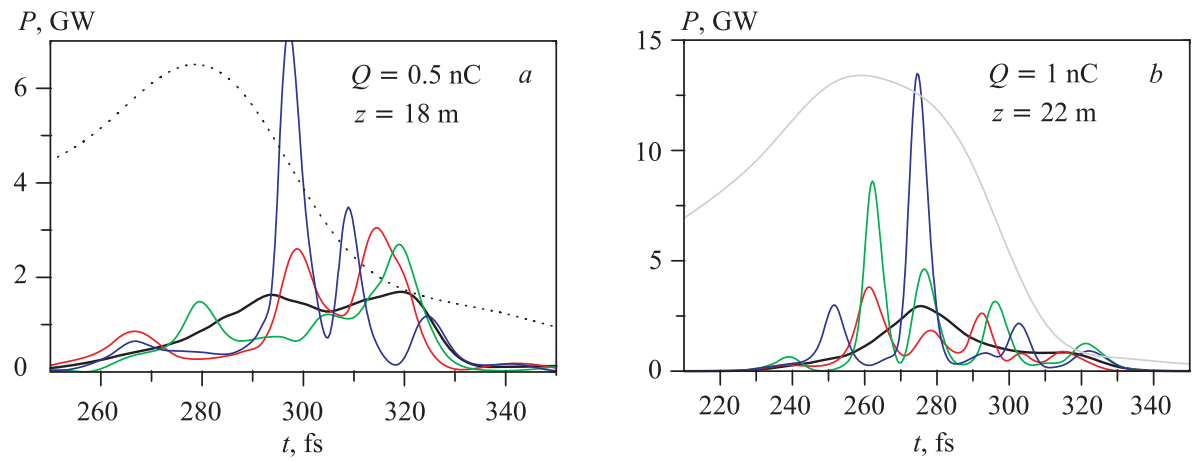

Fig. 4. Radiation power along the bunch for the VUV FEL operating in the saturation regime. $a$ ) Bunch charge is $0.5 \mathrm{nC}, z=18 \mathrm{~m}$. b) Bunch charge is $1 \mathrm{nC}, z=22 \mathrm{~m}$. Thin curves show single shots. Bold curves show averaged profiles. Grey curves show profile of electron bunch
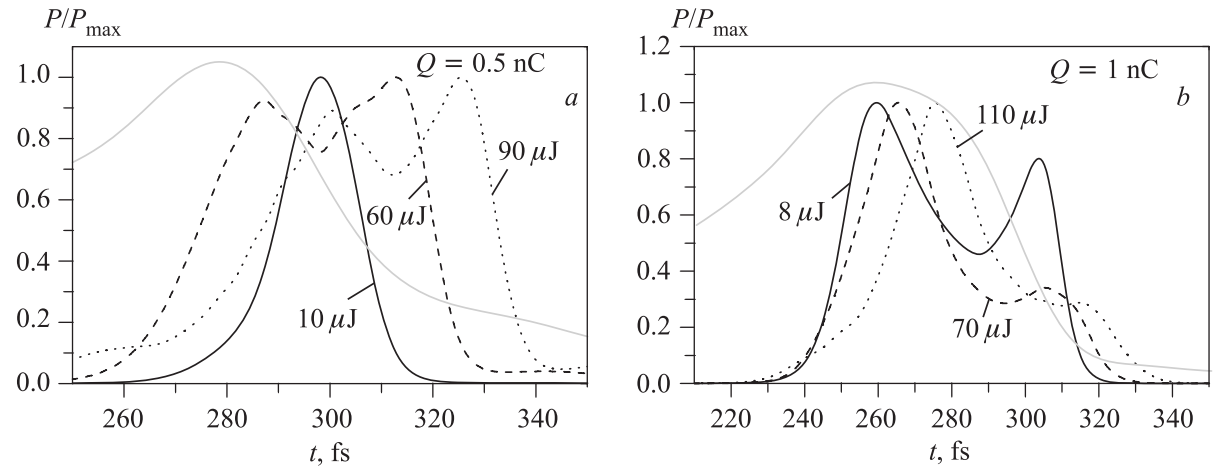

Fig. 5. Evolution of the radiation pulse shape versus average energy in the radiation pulse. Grey line shows electron bunch profile. a) Bunch charge is $0.5 \mathrm{nC}$. b) Bunch charge is $1 \mathrm{nC}$ 
as functions of position along the undulator. One can see that for both charges $(0.5$ and $1 \mathrm{nC})$ saturation is expected in the middle of the undulator for a wavelength of $30 \mathrm{~nm}$.

Expected level of the output energy at saturation is approximately the same, of about $100 \mu \mathrm{J}$, while the saturation length is shorter for the case of $0.5 \mathrm{nC}$. More pronounced difference is in the behavior of the fluctuations of the radiation energy. Larger level of

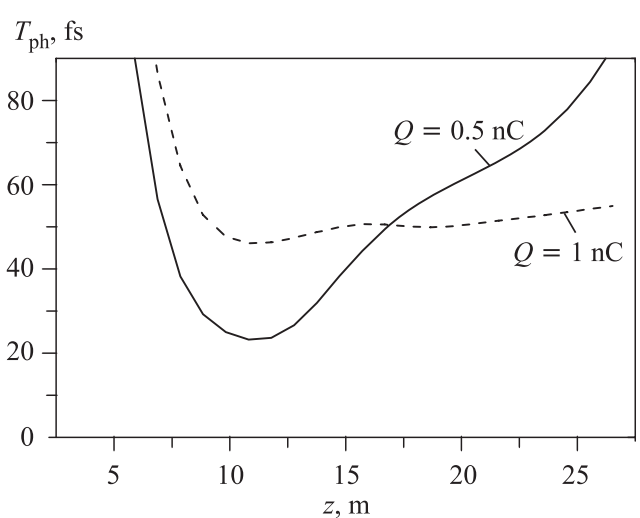

Fig. 6. Evolution of the FWHM radiation pulse length along undulator. Solid and dashed lines correspond to the charge of 0.5 and $1 \mathrm{nC}$, respectively can trace evolution of the radiation pulse envelope from linear regime to the saturation. Figure 6 shows evolution of the FWHM pulse length along the undulator.

For the experiments utilizing the property of ultrashort radiation pulse duration it is important to know the degree of contrast of the radiation pulse. The figure of merit for the degree of contrast is the ratio of the radiation energy within a time window $\tau$ around a spike with maximum peak power to the total energy in the radiation pulse:

$$
C(\tau)=\frac{\int_{-\tau / 2}^{\tau / 2} P(t) d t}{\int_{-\infty}^{\infty} P(t) d t} .
$$

The results for the average degree of contrast shown in Fig. 7 have been derived from 500 statistically independent runs. For fixed time window $\tau$ around the spike with maximum intensity we calculate the value of $C(\tau)$ for each pulse and then perform averaging over ensemble. Solid and dashed curves in Fig. 7 correspond to the end of the linear regime and deep nonlinear regime, respectively. We see that the considered modes of the VUV FEL operation (driven by bunches with 0.5 and $1 \mathrm{nC}$ charge) provide quite different properties for the contrast of the radiation pulse. The VUV FEL driven by $0.5 \mathrm{nC}$ bunch is capable 
of producing short, down to $20 \mathrm{fs}$, radiation pulses with GW-level peak power and contrast of $80 \%$. This happens due to shorter lasing part of the bunch. Maximum contrast of the radiation pulses occurs at the end of the linear mode of operation. Average energy in the radiation pulse is about $10 \mu \mathrm{J}$ in this case. Increase of the radiation energy above this value leads to gradual decrease of the contrast due to radiation pulse lengthening in the nonlinear regime. The case of $1 \mathrm{nC}$ demonstrates the contrast of the radiation pulse nearly independent of the pulse energy: $80 \%$ at 50 fs.
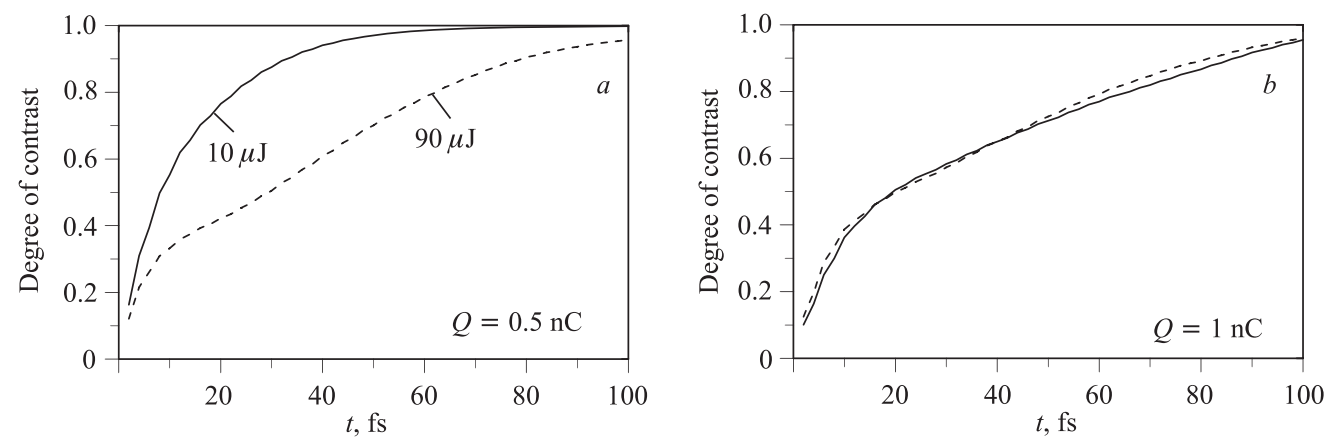

Fig. 7. Degree of contrast versus time gate around a spike with maximum intensity. a) Bunch charge is $0.5 \mathrm{nC} . b$ ) Bunch charge is $1 \mathrm{nC}$. Solid and dashed lines correspond to the case of the linear regime and saturation, respectively

Figure 2 shows average energy in the radiation pulse and rms fluctuations as functions of position along the undulator. Expected level of the output energy at saturation is approximately the same for 0.5 and $1 \mathrm{nC}$ bunch charge, of about $100 \mu \mathrm{J}$, while the saturation length is a little bit shorter for the case of $0.5 \mathrm{nC}$. More pronounced difference is in the behavior of the fluctuations of the radiation energy. Plots presented in Fig. 2 show the value $\sigma_{E}=$ $\sqrt{\left\langle(E-\langle E\rangle)^{2}\right\rangle} /\langle E\rangle$. These fluctuations reach maximum value at the end of the linear regime. It is known that the radiation from SASE FEL operating in the linear regime possesses properties of completely chaotic polarized light $[7,8]$. One important property is that probability distribution of the energy in the radiation pulse must follow gamma distribution:

$$
p(E)=\frac{M^{M}}{\Gamma(M)}\left(\frac{E}{\langle E\rangle}\right)^{M-1} \frac{1}{\langle E\rangle} \exp \left(-M \frac{E}{\langle E\rangle}\right),
$$

where $\Gamma(M)$ is the gamma function, and $M=1 / \sigma_{E}^{2}$. The parameter $M$ can be interpreted as the average number of «degrees of freedom» or «modes» in the radiation pulse. According to Fig. 2 the number of modes for the VUV FEL is expected to be in the range of 3-6. Smaller number of modes for the case of $0.5 \mathrm{nC}$ is a clear indication of shorter pulse duration.

Amplification process in the SASE FEL starts from shot noise in the electron beam, and the radiation pulse initially consists of a large number of longitudinal and transverse modes. In the linear stage of amplification the number of transverse modes in the radiation pulse drops down due to the mode selection process. First, this process passes the stage exponential decay, and then asymptotically approaches of single-mode case. Final degree of transverse coherence 

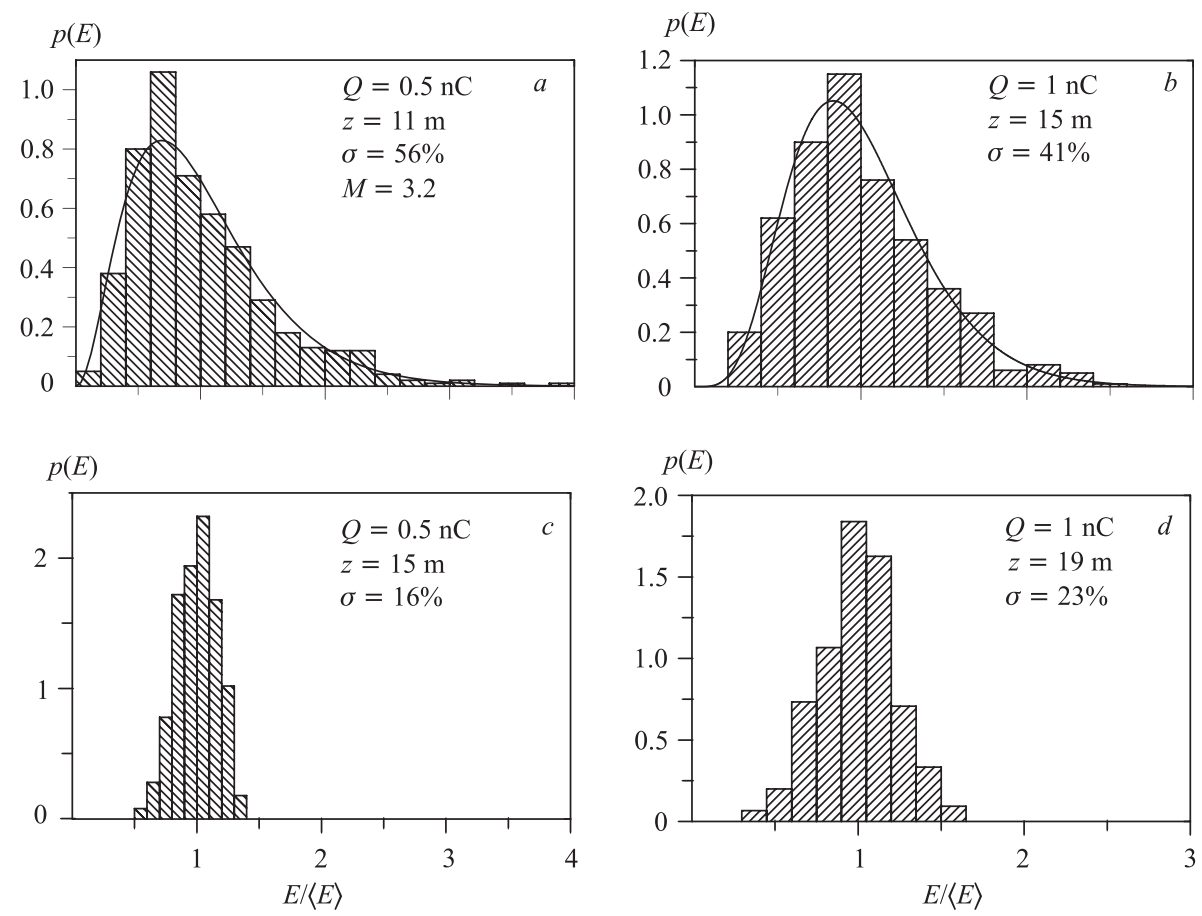

Fig. 8. Probability distributions of the radiation energy at the end of the linear regime (top) and in the deep nonlinear regime (bottom). Left and right columns correspond to the case of 0.5 and $1 \mathrm{nC}$, respectively. Solid lines show gamma distribution

is limited by finite spectrum width of the SASE FEL [9]. The number of longitudinal modes at the end of the linear regime is settled proportionally to the ratio of the length of the lasing part of the bunch to the coherence length. Thus, in the linear regime we have permanent reduction of the number of radiation modes which leads to the growth of the fluctuations of the radiation energy (see Fig. 2).

Fluctuations of the radiation energy change drastically when amplification process enters the nonlinear regime (see Fig. 8). One can see from Fig. 2 that fluctuations of the radiation energy drop down on a scale of about one gain length. Note that such a fast drop of fluctuations is the feature of ultrashort pulse duration [10]. Nature of this phenomenon can be understood by analyzing the structure of the radiation pulse (see Figs. 3 and 4). When amplification process enters nonlinear stage, the group velocity of the radiation becomes to be close to the velocity of light, and saturated wavepackets start to slip forward with respect to the electron bunch. Further growth of the total radiation energy occurs due to the radiation of the bunched electron beam. Since maximum bunching of the electron beam is limited to the unity, this additional radiation is well stabilized, leading to the overall stability of the total energy in the radiation pulse. 


\section{SUMMARY}

Gigawatt-level, laser-like radiation pulses on a sub-50 fs scale are produced in the VUV FEL at DESY with a simple and reliable single-pass SASE FEL scheme. The generation of ultrashort radiation pulses became possible due to specific tailoring of the bunch charge distribution. Such a technique is more reliable and effective with respect to sophisticated HGHG schemes widely discussed as a future alternative to single-pass SASE FEL in terms of shorter pulse duration and better quality of output radiation (see, e.g., [11] and references therein). Key element of the VUV FEL operating in the femtosecond regime is nonlinear bunch compression scheme producing tailored electron bunches with a short high-current leading peak in the density distribution that produces FEL radiation. An important feature of the beam formation system is a strong influence of collective effects. One of them, longitudinal space charge, plays an extremely important role: induced energy chirp along the lasing part of the bunch allows further shortening of the lasing fraction of the electron bunch. As a result, it becomes possible to generate ultrashort, down to $20 \mathrm{fs}$, radiation pulses with GW-level peak power and contrast of $80 \%$.

\section{REFERENCES}

1. Ayvazyan V. et al. // Phys. Rev. Lett. 2002. V.88. P. 104802.

2. Ayvazyan V. et al. // Eur. Phys. J. D. 2002. V. 20. P. 149.

3. Dohlus M. et al. // Nucl. Instr. Meth. A. 2004. V.530. P. 217.

4. Saldin E. L., Schneidmiller E. A., Yurkov M. V. DESY Print TESLA-FEL 2004-06. 2004.

5. Ayvazyan V. et al. // Eur. Phys. J. D. 2006. V.37. P. 297.

6. Saldin E. L., Schneidmiller E. A., Yurkov M. V. // Nucl. Instr. Meth. A. 1999. V.429. P. 233.

7. Saldin E.L., Schneidmiller E.A., Yurkov M.V. The Physics of Free Electron Lasers. Berlin: Springer-Verlag, 1999.

8. Saldin E. L., Schneidmiller E. A., Yurkov M. V. // Opt. Commun. 1998. V. 148. P. 383.

9. Saldin E. L., Schneidmiller E. A., Yurkov M. V. // Opt. Commun. 2000. V. 186. P. 185.

10. Saldin E. L., Schneidmiller E. A., Yurkov M. V. // Nucl. Instr. Meth. A. 2003. V. 507. P. 101.

11. Lambert G. et al. // Proc. of the FEL-2005 Conf. Trieste, Italy, 2004. P. 155. 